\title{
Fatores competitivos de produção em medicina diagnóstica: da área técnica ao mercado
}

Primeira submissão em 14/07/09 Última submissão em 10/08/09 Aceito para publicação em 10/08/09 Publicado em 20/08/09

\section{Production strategic factors in diagnostic medicine: from technical floor to the market}

\author{
Gustavo Aguiar Campana'; Lorena Brito de Faro'; Carmen Paz Oplustil Gonzalez ${ }^{2}$
}

\begin{tabular}{l|l}
\multicolumn{1}{c|}{ unitermos } & res UM \\
Estratégia & $\begin{array}{l}\text { A medicina diagnóstica é, hoje, um conglomerado de especialidades direcionadas à realização de exames } \\
\text { Gestão }\end{array}$ \\
$\begin{array}{l}\text { Produção } \\
\text { realizassem os denominados trade offs entre diferentes fatores estratégicos de produção - qualidade, } \\
\text { Cperação }\end{array}$ & $\begin{array}{l}\text { confiabilidade, flexibilidade, velocidade e custo -, com o objetivo de definir o posicionamento ante a } \\
\text { expectativas dos clientes e de um benchmarking com a concorrência, analisados de forma sistemática, } \\
\text { define o posicionamento estratégico da organização. Para sedimentar os conceitos defendidos neste } \\
\text { artigo, uma simulação de um caso de definição da estratégia da área de produção é apresentada. }\end{array}$
\end{tabular}

\section{abstract}

Diagnostic Medicine is currently a pool of medical specialties oriented to conduct complementary tests for clinical diagnostics. The higher market competitiveness has led management teams to make trade offs among strategic factors such as quality, reliability, flexibility, velocity and costs with the aim to define the contribution of the production area to the company. The determination of these factors based on clients' expectations, benchmarking and subject to systematical analysis define the company strategy. The simulation of a production area strategy planning is presented to demonstrate the concepts discussed in this article.

\section{key words}

Strategy

Management

Production

Operation 
A medicina diagnóstica é, hoje, um conglomerado de especialidades direcionadas à realização de exames complementares no auxílio ao diagnóstico, com impacto nos diferentes estágios da cadeia de saúde: prevenção, diagnóstico, prognóstico e acompanhamento terapêutico. Fazem parte deste mercado os laboratórios de patologia clínica/medicina laboratorial, de anatomia patológica, as clínicas de radiologia e imagem e de outras especialidades, conjuntamente denominados de centro de diagnósticos e também as indústrias de diagnósticos, fornecedores de todas as instituições citadas anteriormente.

A cada ano percebemos tendências à integração destes serviços na então denominada medicina diagnóstica. Tal integração traz benefícios para as diferentes partes relacionadas, como pacientes, que passam a contar com centros de alta resolubilidade; comunidade médica, oferecendo laudos e suporte por meio de diagnósticos integrados; o próprio mercado, que se torna mais competitivo e passa a possuir empresas sólidas com alto poder de investimento na sociedade, favorecendo o crescimento e a profissionalização dos profissionais da saúde; além de incentivar o uso de novas formas de gestão nas empresas de saúde ${ }^{(17)}$. Os diferentes movimentos que demonstram estes novos rumos podem ser observados pelo início da consolidação deste setor, pela verticalização das fontes pagadoras e de prestadores de serviços em diferentes níveis da cadeia e também pela complementaridade da iniciativa privada na prestação dos serviços públicos de saúde.

A cobertura da assistência à saúde no Brasil é, em sua maior parte $(\sim 76 \%)$, realizada pelo setor público por meio do Sistema Único de Saúde (SUS) e financiada pela iniciativa privada ( 54\%). Em dezembro de 2008, o país possuía 40.910.631 beneficiários de planos de saúde (sem plano exclusivamente odontológico) e estes utilizaram, em média, 11,15 exames por beneficiário(1).

No Brasil, o mercado de medicina diagnóstica é bastante pulverizado, composto de cerca de 20 mil empresas, segundo o Cadastro Nacional de Estabelecimentos de Saúde do Ministério da Saúde (CNES/MS).

A atividade das instituições de medicina diagnóstica é pluralizada, com atuação na prestação de serviços, tendo forte impacto na qualidade do atendimento prestado e com foco principal no cliente/paciente; e também como empresa de produtos, fornecendo informação (os laudos) para a comunidade médica. Podemos ainda extrapolar esta informação na geração do conhecimento médico e científico, papel cada vez maior dessas instituições.
Uma mudança significativa é observada no comportamento dos clientes, que começam a fazer prevalecer seu direito de escolha para os serviços de saúde. Mais exigentes, eles passaram a valorizar mais o atendimento em todas as suas fases (agendamento telefônico, recepção, abertura de ficha etc.), a coleta e o pós-venda, assim como a confiança que os médicos depositam nos laboratórios de sua preferência ${ }^{(12)}$.

A maior competitividade do mercado fez com que cada vez mais os gestores iniciassem escolhas do modelo de atuação e estratégias adotadas, acabando por realizar os denominados trade offs - escolhas entre distintas possibilidades. Diferentes fatores e diferenciais competitivos podem ser utilizados neste mercado e devem levar em conta uma variedade bastante ampla de aspectos, como público atendido, mercado regional, concorrentes, fonte pagadora, acionistas, dados demográficos e epidemiológicos etc.

O avanço da tecnologia, assim como a velocidade dessas mudanças, impacta de forma muito clara as empresas e suas áreas de produção, ocasionando importante desigualdade entre as instituições. Contribuem também para esta desigualdade a baixa regulamentação do setor, a grande guerra por preços e a pressão por custos não-relacionada com o aumento na qualidade e na capacitação técnica e profissional das diferentes regiões geográficas, na maioria das vezes associadas à baixa velocidade de disseminação das melhores práticas em saúde ${ }^{(10)}$.

Um mercado competitivo e uma competição saudável (competição "não soma zero") são soluções para a melhoria no sistema de saúde ${ }^{(10)}$.

Muitos fatores ou atributos que tornam a empresa mais competitiva devem partir das áreas de produção, isto é, dos setores responsáveis pela realização dos exames propriamente ditos.

Podemos fazer um paralelo com a engenharia de produção mediante os conceitos de Nigel Slack, definindo a função produção como processos de transformação de produtos ou serviços que satisfazem à necessidade dos clientes (Figura 1) $)^{(14)}$.

A partir desta definição e da Figura 1 podemos observar de forma clara a relação que a função produção exerce com o ambiente, a estratégia das organizações e, principalmente, o efeito direto sobre o consumidor.

As áreas de produção dentro das empresas atuantes no mercado de medicina diagnóstica (na maioria das vezes denominadas de áreas técnicas) são responsáveis 


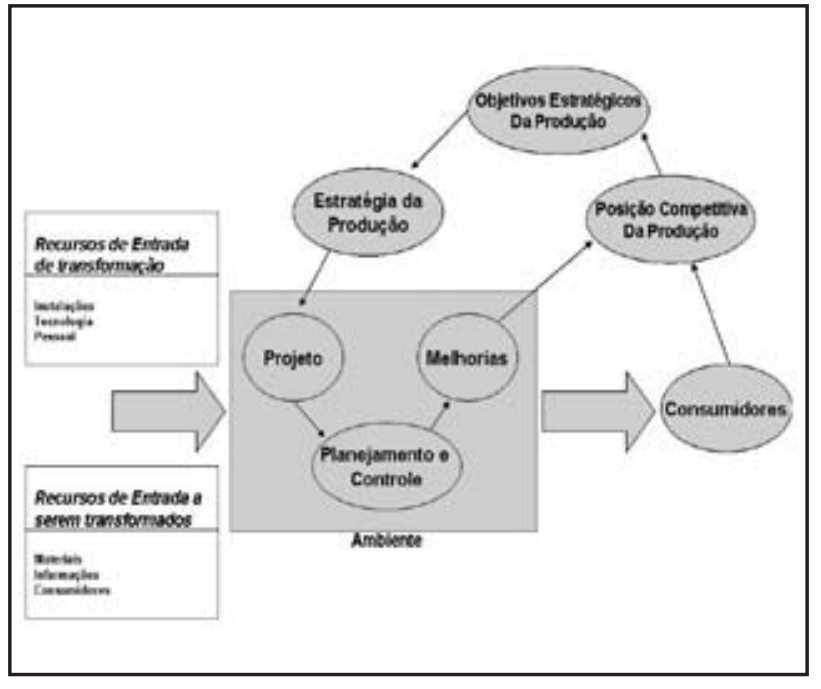

Figura 1 - Função produção(14)

por grande parte dos ativos instalados e dos gastos existentes, chegando a consumir, em média, 25\% a 35\% da receita líquida gerada.

Para posicionar a função produção como um diferencial para a organização é necessária a definição da estratégia de produção a ser adotada, ou, ainda, definir qual é o rumo da área de produção e como esta contribuirá para a organização.

Segundo Hayes e Wheelwright, podemos classificar a função produção ao longo do tempo em quatro diferentes estágios de contribuição em relação à organização em que ela está inserida, como mostra a Figura $\mathbf{2}^{(4)}$.

Em cada uma das etapas, a área de produção apresenta um propósito específico:

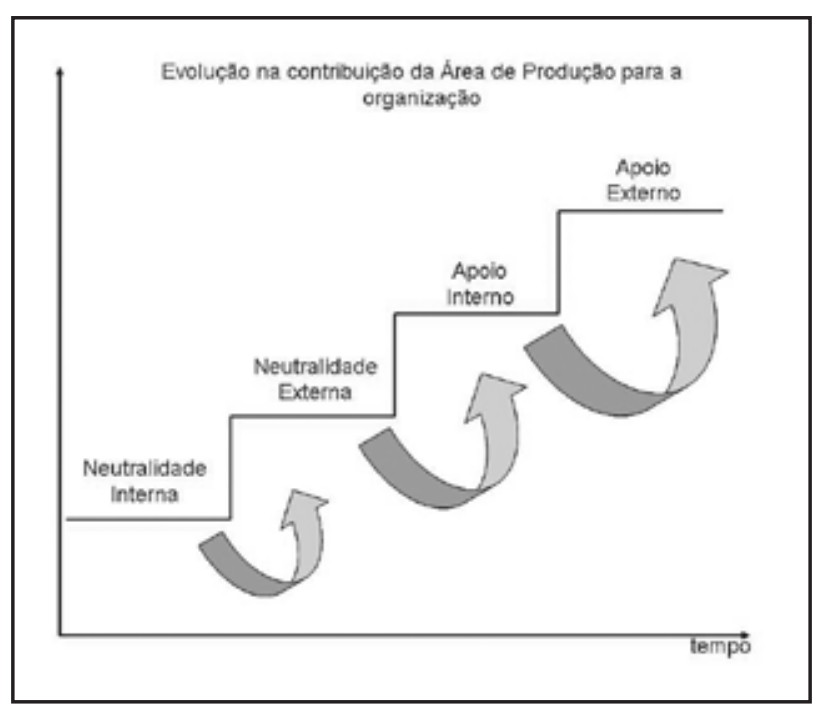

Figura 2 - Evolução na contribuição da área de produção
- neutralidade interna: expressa o conceito de "parar de atrapalhar a organização", ou "parar de cometer erros";

- neutralidade externa: nesta fase, a produção passa a ter seu desempenho analisado em relação à de outras organizações. Posiciona a empresa de forma a querer obter um posicionamento de "estar entre as melhores do setor", pela busca constante de melhorias;

- apoio interno: neste caso, a empresa já se posicionou entre as "melhores do setor" e apresenta uma visão clara dos objetivos estratégicos que a dirigem. Nesta fase, a área técnica já é o motivo de captação do cliente, seja no mercado ambulatorial ou, mais ainda, no mercado de apoio laboratorial (relacionamento entre empresas). Conquistar o cliente por atributos competitivos da função produção é uma realidade para o mercado de medicina diagnóstica que se encontra nesta etapa;

- apoio externo: é a melhor do setor. A empresa apresenta, como diferenciais, "superioridade, inovação e referência". Identificar tendências, lançar novos produtos e disseminar conhecimento são pontos de destaque das operações que atuam como apoio externo às instituições. Neste caso, não só suporta as vendas, mas é o principal atrativo e o valor agregado do produto ou serviço, participando, assim, da definição das estratégias corporativas.

Com a definição do posicionamento diante da contribuição da área de produção para a organização, é fundamental determinar como alcançar os objetivos e as metas propostas. Nesta etapa devem ser definidas formas e ferramentas de trabalho que suportarão as atividades, e devem ser colocadas de forma clara ao mercado (meio externo), além da operação (meio interno), dos fatores competitivos, dos pilares de atuação e dos diferenciais que serão oferecidos.

Nigel Slack define como fatores de competição para as áreas de produção: qualidade, confiabilidade, flexibilidade, velocidade e custo ${ }^{(14)}$.

Fazendo um paralelo do conhecimento já difundido pela engenharia de produção com a realidade das operações de medicina diagnóstica, posicionamos cada um dos fatores mencionados anteriormente de forma a tentar descrever essa relação.

\section{Qualidade}

A qualidade técnica em medicina diagnóstica é condição básica de permanência no mercado, ou seja, permite que as empresas compitam dentro de um nicho de atuação, não sendo apenas mais um diferencial de 
serviço. Uma empresa de medicina diagnóstica que não possui qualidade técnica está excluída do mercado pelos seus próprios clientes - pacientes, operadoras de saúde, comunidade médica etc.

Como um atributo competitivo de produção, podemos definir a qualidade como "fazer certo da primeira vez" ou, então, "manter os processos sob controle". Isto se aplica a rotinas técnicas (repetições, por exemplo), controles internos de qualidade, processos de suporte etc. Para o mercado, aumenta o grau de confiança na empresa e, para a operação, melhora os processos internos de produção e impacta positivamente os outros fatores, como velocidade de produção, custo e confiabilidade.
Destacamos agora a importância do controle da operação em nosso setor. Diversos autores de patologia clínica/ medicina laboratorial demonstram que cerca de $40 \%$ dos erros ocorrem na fase pré-analítica, $7,3 \%$ a $10 \%$ na fase analítica e $45 \%$ a $47,2 \%$ na pós-analítica( ${ }^{(7)}$.

A qualidade, como padronização e controle, dá-se por meio de constante observação e análise de indicadores de controle. Recentemente uma publicação propôs uma forma de padronização destes indicadores, a fim de definir um alinhamento entre as práticas nas diferentes empresas ${ }^{(18)}$ (Tabela 1).

Atualmente, diferentes modelos de acreditação de toda a gestão da qualidade endossam este atributo nas

Tabela 1

Indicadores de qualidade em medicina laboratorial (por estágios do processo total do exame)

Solicitação do exame
Índice de solicitação de amostras inapropriadas
Processo de coleta de amostras
Satisfação do paciente na coleta
Índice de hematomas
Índice de solicitação de novas coletas
Contaminação de hemocultura
Identificação da amostra, preparação e transporte
Índice de rejeição das amostras
Erro na informação da amostra (CB*)
Desempenho em teste de proficiência
Discrepância entre citologia e biópsia ginecológica
\% ClQ e testes com CV > máximo definido
\% de exames alterados
MTTR
MTBF
\% de repetição de exames

Adaptado de "Laboratory medicine quality indicators". AJCP, 2009(6).

CB: código de barras; CIQ: controle interno de qualidade; CV: coeficiente de variação; MTTR: tempo médio para reparo; MTBF: tempo médio entre falhas;

TAT: turnaround time. 
empresas de medicina diagnóstica e alinham os processos de produção com as melhores práticas dos mercados nacional e internacional. A presença de diferentes metodologias e órgãos acreditadores permite o acesso a esta prática para as empresas de qualquer porte. Podemos citar como exemplo órgãos nacionais, como o Programa de Acreditação de Laboratórios Clínicos (PALC) ${ }^{(15)}$, da Sociedade Brasileira de Patologia Clínica/Medicina Laboratorial (SBPC/ML), e a Organização Nacional de Acreditação $(\mathrm{ONA})^{(9)}$, e internacionais, como os programas do College of American Pathologists (CAP) ${ }^{(3)}$ e o Joint Comission $(\mathrm{JCl})^{(16)}$.

Em 1999, a Associação Mundial das Sociedades de Patologia e Medicina Laboratorial (WASPaLM) e a Federação Internacional de Química Clínica e Medicina Laboratorial (IFCC) publicaram uma declaração sobre acreditação de laboratórios clínicos referindo que "é do interesse dos pacientes, da sociedade e do governo que os laboratórios clínicos operem dentro de altos padrões de competência profissional e técnica devida"(3):

- as decisões quanto a diagnóstico, prognóstico e terapêutica são, frequentemente, baseadas nos resultados ou na interpretação de exames laboratoriais, portanto danos irreversíveis podem ser causados por resultados errôneos;

- os usuários de serviços de laboratórios, tanto pacientes quanto médicos, podem não possuir conhecimentos técnicos suficientes para avaliar se um laboratório está operando em um nível satisfatório de qualidade;

- os pacientes e, em menor grau, os médicos, podem não ter opção quanto a qual laboratório utilizar;

- os exames de laboratório podem ser dispendiosos e os pacientes, as seguradoras, ou o governo, que pagam os exames, têm o direito de esperar que o laboratório forneça informações válidas;

- é do interesse dos laboratórios que sua competência seja atestada por processo de auditoria, por comparação com padrões apropriados e que isto se torne público.

\section{Confiabilidade}

Como confiabilidade descrevemos o ato de realizar e entregar ao cliente aquilo que foi previamente acordado: o exame solicitado por meio de uma metodologia determinada dentro de um prazo de entrega contratado.

A confiabilidade da operação aumenta o grau de confiança do mercado para com a empresa, pois entregamos aos nossos clientes exatamente o produto ou serviço com que nos comprometemos e pelos quais estamos sendo remunerados. A insatisfação dos clientes gerada por atrasos em diversos processos da cadeia de saúde já foi descrita, assim como o julgamento das instituições de medicina diagnóstica é também baseado na velocidade e/ou entrega de resultados ${ }^{(6)}$. A confiabilidade pode ser traduzida como a avaliação do produto ou serviço no momento do seu uso.

Quando tratamos de laboratórios que atendem a prontos-socorros, este diferencial é prioridade para um bom atendimento. Nestas situações, cerca de $60 \%$ das informações de prontuários médicos são relacionados com exames laboratoriais, concluindo que atrasos nos resultados causam atrasos em diagnóstico e tratamento(5).

\section{Flexibilidade}

Flexibilidade de produção em medicina diagnóstica pode ser vista como a facilidade (e disposição) para alterações em rotinas de exames, metodologias e adequação da capacidade instalada de produção às mudanças de demanda, ou seja, é a capacidade de adaptação da empresa ou sua disponibilidade para mudanças. É permitir que os clientes e o mercado (científico) direcionem o modo de produção, e não que sejam obrigados a aceitar o que entendemos como necessário. Podemos ainda classificar este atributo como flexibilidade de produto, de mix (menu de exames), de volume ou de entrega.

Uma constante mudança observada em medicina laboratorial é a entrada de novas tecnologias no mercado. A introdução de metodologias como biologia molecular, espectrometria de massa, cromotografia líquida de alta performance, entre outras na rotina dos laboratórios, é um exemplo claro da grande transformação necessária para acompanharmos a tecnologia do mercado. Destacamos também a necessidade de renovação dos tipos de produtos ofertados ao mercado, retirando de rotinas os testes obsoletos. As principais influências quanto à evolução dos tipos de testes laboratoriais já foram reportadas, tais como regulamentações, remuneração, mudanças na prática médica, tecnologia diagnóstica, custos e mercado(7). A Agência Nacional de Saúde (ANS) definiu em 2008 o rol de procedimentos que obrigatoriamente devem ser cobertos pelas operadoras de saúde privada. Este novo rol contempla cerca de 2.900 procedimentos. Nos EUA, um estudo realizado em 1998, com um número significativo de laboratórios, demonstrou que 55\% deles haviam descontinuado algum 
tipo de exame nos últimos dois anos, sendo $75 \%$ dos casos devidos a mudanças de tecnologia, seguidas por fatores de regulamentação. Ainda, 39\% das empresas haviam incluído pelo menos algum teste no menu praticado(7).

As transformações decorrentes de alterações do perfil de pacientes e da sociedade, como, por exemplo, enveIhecimento da população, aumento na complexidade da tecnologia médica, maior prevalência de doenças crônicas, mercado (sistema) pouco organizado e evolução da tecnologia da informação, fazem da flexibilidade ponto importante de diferenciação no mercado(11).

\section{Velocidade}

A velocidade de processamento traduz-se para o meio externo em prazo de entrega de resultados e para o meio interno em processos eficientes e enxutos. O impacto de resultados rápidos e prazos reduzidos é positivo para a saúde pública, para uma atuação médica de maior qualidade; assim como é também um diferencial em atendimento e serviço ao cliente ${ }^{(20)}$. Destacamos também a necessidade de observar a velocidade de processamento e entrega de resultados de exames sob a ótica do cliente médico, e não por meio apenas de benchmarking de mercado $^{(6)}$.

Em alguns setores deste mercado, como o business-tobusiness, serviços entre empresas, este é um dos atributos de maior importância para os clientes.

Atualmente, um dos indicadores mais utilizados em medicina laboratorial é o turnaround time (TAT), ou tempo total de realização do exame do atendimento ao cliente à liberação do resultado. A Tabela 2 demonstra diversas atividades em diferentes fases do processo que auxiliam na redução do TAT.

\section{Custo}

Operar com custos reduzidos é fundamental para que a empresa obtenha resultados satisfatórios que permitam que ela responda às partes relacionadas (stakeholders) de forma positiva - seja com geração de empregos, investimentos, compras de produtos e serviços, relações institucionais, trabalhos sociais ou dividendos.

A queda da remuneração por exames é uma realidade observada nos últimos anos, e os laboratórios cada vez mais se adaptam a este novo cenário por meio de mudanças de modelos de negócio, automação, corte de custos de mão-de-obra, otimização de ativos e processos etc. A realização de custeio e acompanhamento dos custos de produção, além de análises sistemáticas de demanda e mix de exames (menu e variedade realizada), que impactam de forma significativa a viabilidade dos laboratórios, é essencial para a avaliação econômico-financeira das instituições(2).

A pressão continua por redução de custos é não só uma realidade, mas uma tendência em medicina diagnóstica, e é o principal catalisador dos processos de consolidação do setor $^{(8)}$. Uma operação de baixo custo de produção pode ser convertida em margem operacional ou em redução de preço de venda.

A seguir, exemplificamos o impacto nos meios externo e interno de cada um destes fatores (Tabela 3).

Está clara a necessidade de um posicionamento estratégico alinhado com os fatores estratégicos operacionais. As empresas de medicina diagnóstica se diferenciam em produtos e serviços dependendo de seu público-alvo, mercado de atuação, possibilidade de investimentos, expectativas de retorno e nicho de atuação.

Realizar um trade-off (escolha) não exclui a utilização de outros fatores, ou, ainda, muitos atributos impactam de forma positiva qualquer um dos fatores de competitividade, como, por exemplo, certamente uma operação de alta velocidade significa processos enxutos, o que acarreta baixos custos de operação.

Mais importante é olhar o mercado e seus players para mapear oportunidades e "espaços em branco" como ferramenta de auxílio no posicionamento estratégico da organização.

Analisando o impacto no cliente de cada um dos cinco fatores discutidos, devemos determinar para cada um deles um objetivo de desempenho. Hill descreve esta classificação em três diferentes classes, conforme a Tabela $3^{(4)}$.

Uma vez determinados os objetivos de desempenho para os clientes e a classe para os fatores, a performance da empresa nestes mesmos atributos deve ser determinada em comparação com a concorrência, respondendo à pergunta:" como estamos em relação à concorrência?". As possíveis respostas são:

- melhor que a concorrência;

- igual à concorrência;

- pior que a concorrência. 


\begin{tabular}{|c|c|c|}
\hline Tabela 2 & Desempenho do TAT & \\
\hline Estágio & Elemento & Ações para melhora do TAT \\
\hline \multirow{8}{*}{ Fase pré-analítica } & Pedido médico & $\begin{array}{l}\text { Nomenclatura padrão } \\
\text { Telas padronizadas no sistema } \\
\text { Solicitação eletrônica de exames }\end{array}$ \\
\hline & Informação apropriada & Assegurar a acurácia da transmissão de dados \\
\hline & Coleta & Inspecionar as práticas utilizadas \\
\hline & Identificação das amostras & Utilizar código de barras \\
\hline & Entrega das amostras & Considerar tubo pneumático, automação, esteiras etc. \\
\hline & Tipo de amostra & Revisar o uso de plasma, soro e sangue total \\
\hline & Recepção de amostras & Utilizar leitor de código de barras \\
\hline & $\begin{array}{l}\text { Separação/distribuição de } \\
\text { amostras }\end{array}$ & $\begin{array}{l}\text { Automação pré-analítica: separação, centrifugação, } \\
\text { destampadores, aliquotadores etc. } \\
\text { Utilizar tubo primário }\end{array}$ \\
\hline \multirow{6}{*}{ Fase analítica } & & Considerar automação \\
\hline & & Avaliar throughput - capacidade por tempo \\
\hline & Equipamentos & Assegurar o mínimo de downtime - parada de equipamentos \\
\hline & & - e prever Dackup \\
\hline & & Considerar autoverificação de resultados \\
\hline & Controle de qualidade & Procedimentos eficientes \\
\hline \multirow{6}{*}{ Fase pós-analítica } & \multirow{6}{*}{ Liberação de laudos } & Interfaceamento e liberação automática \\
\hline & & Utilizar laudos preliminares \\
\hline & & Liberação de resultados por diversas vias: internet, PDA, \\
\hline & & pager, SMS, broadcast etc. \\
\hline & & Impressão automática por localização (ex.: UTI) \\
\hline & & $\begin{array}{l}\text { Oferecer assessoria na interpretação (helpdesk, testes } \\
\text { reflexos etc.) }\end{array}$ \\
\hline \multirow{3}{*}{ Todas as fases acima } & & $\begin{array}{l}\text { Acompanhar o TAT e buscar melhoria contínua (média, } \\
\text { mediana, benchmarkin) }\end{array}$ \\
\hline & & Avaliar os fluxos dos processos \\
\hline & & Eliminar erros e desperdícios \\
\hline
\end{tabular}

Desempenho do TAT: adaptado de Laboratory Results. AJCP, 2000.

TAT: turnaround time; PDA: assistente pessoal digital; SMS: serviço de mensagens curtas; UTI: unidade de terapia intensiva.

Com essas definições, aos olhos dos clientes e da concorrência, estratificando em uma escala de 1 a 9, temos, portanto, formada uma matriz de importância/desempenho que auxiliará na tomada de decisão e, principalmente, na priorização de ações (Figura 3) (14).

O desdobramento de todo este trabalho deve ser feito por meio de planos de ação detalhados, com objetivo, responsáveis, cronogramas e indicadores. A matriz importância/desempenho deve ser reavaliada de tempos em tempos e encarada de forma dinâmica, assim como o mercado e as expectativas dos nossos clientes.

\section{Um caso hipotético}

Para sedimentar os conceitos colocados neste artigo, vamos simular um caso de definição da estratégia da produção. 


\begin{tabular}{|c|c|c|}
\hline \multirow{2}{*}{$\frac{\text { Tabela } 3}{\text { Meio interno }}$} & \multicolumn{2}{|c|}{$\begin{array}{l}\text { Impacto de cada fator nos meios } \\
\text { externo e interno }\end{array}$} \\
\hline & Fator & Meio externo \\
\hline $\begin{array}{l}\text { Processos } \\
\text { certos }\end{array}$ & Qualidade & $\begin{array}{l}\text { Qualificação para } \\
\text { o mercado }\end{array}$ \\
\hline & & Referência \\
\hline $\begin{array}{l}\text { Processos } \\
\text { certos }\end{array}$ & Confiabilidade & Maior confiança \\
\hline Padronização & & $\begin{array}{l}\text { Entrega do que } \\
\text { foi acordado }\end{array}$ \\
\hline $\begin{array}{l}\text { Adaptação às } \\
\text { mudanças }\end{array}$ & Flexibilidade & Customização \\
\hline & & Adaptação \\
\hline $\begin{array}{l}\text { Processos } \\
\text { rápidos e } \\
\text { enxutos }\end{array}$ & Velocidade & Entregas rápidas \\
\hline $\begin{array}{l}\text { Alta } \\
\text { produtividade }\end{array}$ & Custo & Preço baixo \\
\hline Margem alta & & Competitividade \\
\hline
\end{tabular}

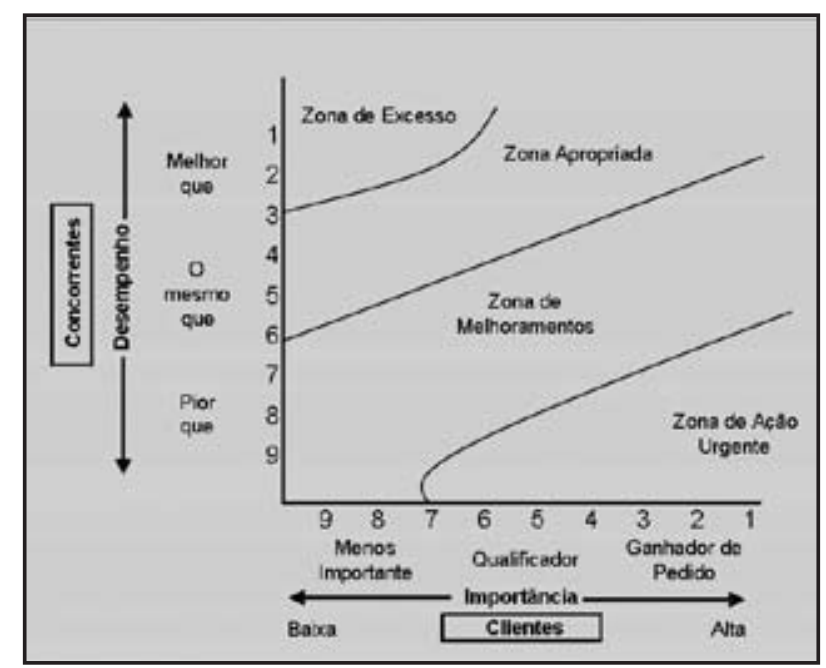

Figura 3 - Matriz de importância/desempenho Adaptada de Slach, N. ${ }^{(5)}$

O gestor de um laboratório de patologia clínica/medicina laboratorial atuante no mercado de apoio nacional decide definir a estratégia de produção de sua empresa. Para isso convoca uma reunião com diferentes líderes e colaboradores dos mais diversos setores: áreas de produção, comercial, marketing, suprimentos, qualidade, atendimento ao cliente e assessoria médica.
Depois da apresentação dos conceitos demonstrados neste artigo, iniciam-se discussões sobre a classificação de cada um dos fatores e a determinação da posição da empresa em cada um deles ante seus clientes e em comparação com seus concorrentes. Neste momento é importante observar a variedade de entendimento e as impressões para cada um dos setores: o pessoal de vendas preocupa-se muito com o custo, para transformá-lo em melhores preços; o pessoal de atendimento ao cliente, com confiabilidade, e assim por diante.

Uma vez determinada esta classificação, os dados encontrados foram plotados na matriz de importância/desempenho, obtendo o cenário descrito na Figura 4.

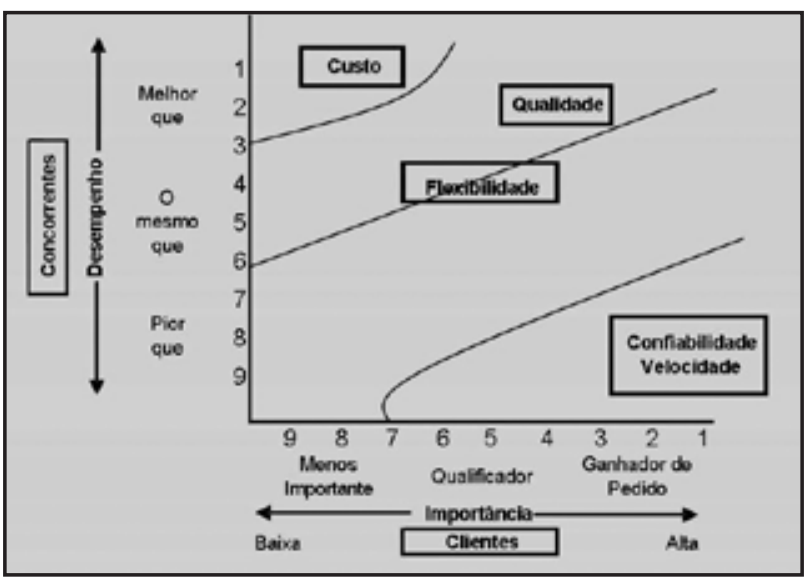

Figura 4 - Matriz de importância/desempenho no mercado de apoio Adaptada de Slach, N.(5)

Observamos então que temos dois importantes fatores ganhadores de pedidos em que estamos piores que a concorrência: confiabilidade e velocidade. Isto porque nossos concorrentes atrasam menos os resultados de exames e fornecem prazos mais curtos de liberação, ambos fatores que captam cliente no mercado de apoio, onde nosso laboratório hipotético está inserido.

Com toda esta análise, a discussão passa a ter um foco em ações de melhoria priorizando aquelas de necessidade urgente de mudança. Um plano de ação é então formulado detalhadamente para a implementação da nova estratégia.

Os possíveis resultados destas análises são extremamente abrangentes e posicionam a organização e sua marca no mercado. Existem empresas que se pautam pela estratégia da flexibilidade, inserindo sempre novos produtos no mercado, assim como outras que optam pela velocidade como o seu principal diferencial, entregando seus resultados com 
Tabela 4

Classes Objetivo de desempenho da produção

Ganhadores Influencia o nível e a qualidade de de pedido pedidos

Qualificadores É considerado pelos clientes quando de pedido está acima de um determinado nível Menos Não são considerados pelos clientes importantes um fator de decisão maior rapidez que a concorrência. As instituições de referência no mercado posicionam-se pelo atributo qualidade; e aquelas que buscam escala, pelo diferencial de custo.

Cada empresa deve definir sua estratégia para buscar atender o mercado e seu cliente.

Com isso, demonstramos que as estratégias definidas para a função produção das organizações de medicina diagnóstica podem ser o diferencial das empresas e posicioná-las estrategicamente.

\section{Referências}

1. AGÊNCIA NACIONAL DE SAÚDE (ANS) Caderno de Informação da Saúde Suplementar - Março 2009. Disponível em: <http://www.ans.org.br>.

2. ASH, K. O. Impact of cost cutting on Laboratories: new business strategies for laboratories. Clin Chem, v. 42, n. 5, p. 822-6, 1996.

3. COLLEGE OF AMERICAN PATHOLOGISTS (CAP). Disponível em: <http://www.cap.org>.

4. CORRÊA, H.; CORRÊA, C. Administração de produção e de operações. São Paulo: Ed. Compacta, Atlas, 2005.

5. HOLLAND, H. et al. Reducing laboratory turnaround time outliers can reduce emergency department patient length of stay. Am J Clin Pathol, v. 124, n. 5, p. 672-74, 2005.

6. HOWANITZ, J.; HOWANITZ, P. Laboratory results. Am J Clin Pathol, v. 116, n. 3, p. 311-5, 2001.

7. LaBEAU, K. M. et al. Clinical laboratory test menu changes in the Pacific Northwest: 1994 to 1996. Clin Chem, v. 44, n. 4, p.833-8. 1998.

8. MARKIN, R.; WHALEN, S. Laboratory automation: trajectory, technology and tatics. Clin Chem, v. 46, n. 5, p. 764-71, 2000.

9. ORGANIZAÇÃO NACIONAL DE ACREDITAÇÃO (ONA). Disponível em: <http://www.ona.org.br>.

10. PORTER, M.; TEISBERG, E. Redefining competition in health care. Harvard Business Review, 2004. p. 1-16.
11. PRICE, C.; CHRISTENSON, R. Evaluating new diagnostic technologies: perspectives in the UK and US. Clin Chem, v. 54, n. 9, p. 1421-23. 2008.

12. SEKI, M. et al. A inovação de valores no laboratório clínico. J Bras Patol Med Lab, v. 39 n. 3, p. 211-4, 2003.

13. SHAHRAM, S.; SUSAN, S. Laboratory Medicine Quality Indicators. Am J Clin Pathol, v. 131, n. 3, p. 418-31, 2009.

14. SLACK, N. et al. Administração da produção. 2. ed. Atlas, 2002.

15. SOCIEDADE BRASILEIRA DE PATOLOGIA CLÍNICA/ MEDICINA LABORATORIAL (SBCP/ML). Disponível em: <htpp://www.sbpc.org.br>.

16. THE JOINT COMMISSION (JCl). Disponível em: <htpp:// www.jointcommission.org>.

17. VETTORI, E. A saúde sob pressão. Revista Mundo Corporativo, v. 4, n. 13, p. 19-21, 2006.

18. WESTGARD, J. The quality of laboratory testing today. Am J Clin Pathol, v. 125, n. 3, p. 343-54, 2006.

19. WORLD ASSOCIATION OF SOCIETIES OF PATHOLOGY AND LABORATORY MEDICINE (WASPALM). Disponível em: <http://www.waspalm.org>.

20. ZARBO, R.; NAKHLEH, R.; WALSH, M. Customer satisfaction in anatomic pathology. Arch Pathol Lab Med, v. 127, n. 1, p. 23-9, 2003. 